\title{
Rank-width and Well-quasi-ordering
}

\author{
Sang-il Oum \\ Program in Applied \& Computational Math. \\ Princeton Univ.
}

November 15, 2004 


\section{Introduction}

- Cut-rank function

- Rank-decomposition and Rank-width

- Clique-width

- Well-quasi-ordering 


\section{Cut-Rank Function}

- $G$ : graph.

- $(A, B)$ : partition of $V(G)$.

Let $M_{A}^{B}(G)=\left(m_{i j}\right)_{i \in A, j \in B}$ be a $A \times B$ matrix over $\mathrm{GF}(2)$ such that

$$
m_{i j}= \begin{cases}1 & \text { if } i \text { is adjacent to } j \\ 0 & \text { otherwise. }\end{cases}
$$

Def: Cut-rank $\operatorname{cutrk}_{G}(A)=\operatorname{rank}\left(M_{A}^{B}(G)\right)$.

Prop. cutrk $_{G}$ is symmetric submodular, i.e.

$$
\begin{gathered}
\operatorname{cutrk}_{G}(X)+\operatorname{cutrk}_{G}(Y) \geq \operatorname{cutrk}_{G}(X \cap Y)+\operatorname{cutrk}_{G}(X \cup Y) \\
\operatorname{cutrk}_{G}(X)=\operatorname{cutr}_{G}(V(G) \backslash X)
\end{gathered}
$$




\section{Rank-decomposition and Rank-width}

Def. - Rank-decomposition of $G:(T, \mathcal{L})$. Cubic tree $T$, bijection $L: V \rightarrow\{x: x$ is a leaf of $T\}$.

- Width of $(T, \mathcal{L})$ :

$$
\max _{e \in T} \operatorname{cutrk}_{G}\left(A_{e}\right)
$$

where $\left(A_{e}, B_{e}\right)$ is a partition of $V(G)$ induced by $e \in T$.

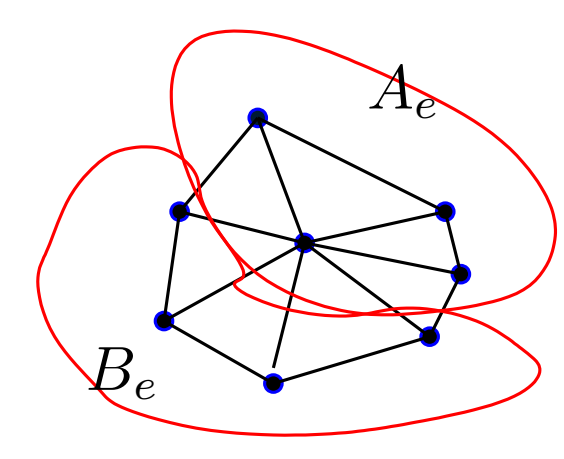

G g

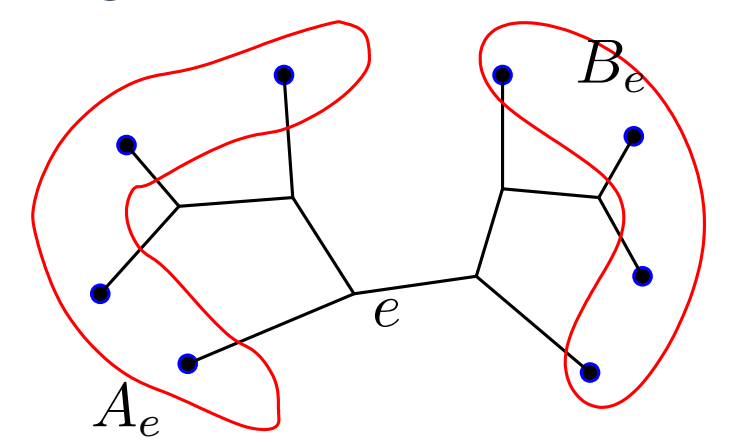

Rank-decomposition of $G$

$\operatorname{rank}\left(\begin{array}{cccc}1 & 0 & 0 & 0 \\ 0 & 0 & 0 & 0 \\ 1 & 1 & 1 & 1 \\ 0 & 0 & 0 & 1\end{array}\right)$

- Rank-width of $G$, denoted by $\operatorname{rwd}(G)$ : minimum width over all possible rank-decompositions of $G$ [Oum and Seymour, 2004] 


\section{Rank-width and Clique-width}

- Clique-width: defined by [Courcelle and Olariu, 2000]

- (Rank-width and Clique-width are compatible)[Oum and Seymour, 2004]

$$
\text { rank-width } \leq \text { clique-width } \leq 2^{\text {rank-width+1 }}-1
$$

- Many NP-hard problems are solvable in polynomial time, if an input is restricted to graphs of bounded clique-width.

Let $C$ be a set of graphs. We ask; " $\exists$ an alogrithm that, for every ??? formula $\varphi$, answers whether there exists $G \in C$ such that $\varphi(G)$ is true".

- (Seese's conjecture [Seese, 1991]) every MSOL formula on graphs is decidable on $C$. (open) $\Rightarrow$ Bounded clique-width

- ([Courcelle and Oum, 2004]) every MSOL formula with Even $(X)$ predicate on graphs is decidable on $C$. $\Rightarrow$ Bounded clique-width 


\section{Well-quasi-ordering}

- $\leq$ is a quasi-ordering if reflexive $(a \leq a)$ and transitive $(a \leq b, b \leq c \Rightarrow$ $a \leq c)$.

- A quasi-ordering $\leq$ on $X$ is a well-quasi-ordering if for every infinite sequence $x_{1}, x_{2}, \ldots$ in $X$,

$$
\exists i<j \text { such that } x_{i} \leq x_{j} .
$$

In other words, $X$ is well-quasi-ordered by $\leq$.

Equivalently, every infinite sequence in $X$ contains an infinite nondecreasing subsequence.

- Examples:(well-quasi-ordered) A set of positive integers with $\leq$. Any finite set. Finite trees with graph minor (Kruskal's theorem)

- Examples: (not well-quasi-ordered) A set of integers with $\leq$. 


\section{Graphs of Bounded Rank-width are well-quasi-ordered}

WANTED: an appropriate quasi-ordering on graphs 


\section{Induced Subgraph Relation is not enough}

- Say $G_{1} \leq G_{2}$ if $G_{1}$ is isomorphic to an induced subgraph of $G_{2}$.

- $C_{n}$ : a cycle of length $n$.

- Consider $X=\left\{C_{3}, C_{4}, C_{5}, \ldots\right\}$.

- $X$ has bounded rank-width (at most 4).

- no $C_{i}$ is an induced subgraph of $C_{j}(i \neq j)$.

Note that if $H$ is an induced subgraph of $G$, then clique-width of $H \leq$ clique-width of $G$, rank-width of $H \leq$ rank-width of $G$.

It would be nice if a set of graphs of bounded rank-width is closed under $\leq$. (So the graph minor is not appropriate!) 


\section{Local Complementation \& Vertex-Minor}

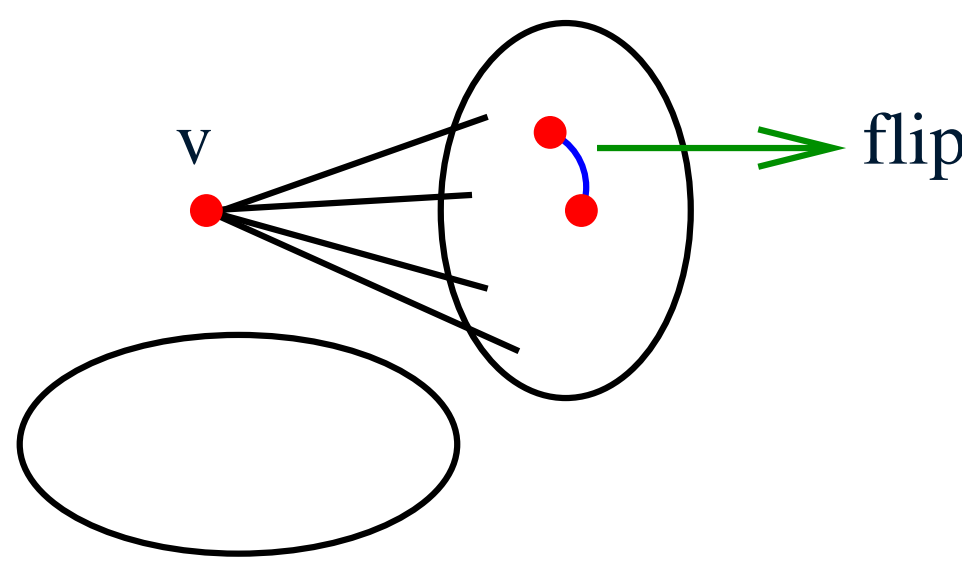

$G * v$
- $G * v$ and $G$ have the same cut-rank function.

- $G$ is locally equivalent to $H$ if $H=G * v_{1} * v_{2} * \cdots v_{k}$.

- Call $H$ is a vertex-minor of $G$, if $H$ can be obtained by a sequence of local complementations and vertex deletions.

- $G * v$ and $G$ have the same rank-width.

- Therefore, if $H$ is a vertex-minor of $G$, then

$$
\text { rank-width of } H \leq \text { rank-width of } G \text {. }
$$




\section{Statement of our thm}

Thm. If $\left\{G_{1}, G_{2}, \ldots\right\}$ is an infinite sequence of graphs of rank-width $\leq k$, then there exists $i<j$ such that

$G_{i}$ is isomorphic to a vertex-minor of $G_{j}$.

In fact, we prove a stronger theorem.

Thm. If $\left\{G_{1}, G_{2}, \ldots\right\}$ is an infinite sequence of graphs of rank-width $\leq k$, then there exists $i<j$ such that

$G_{i}$ is isomorphic to a pivot-minor of $G_{j}$.

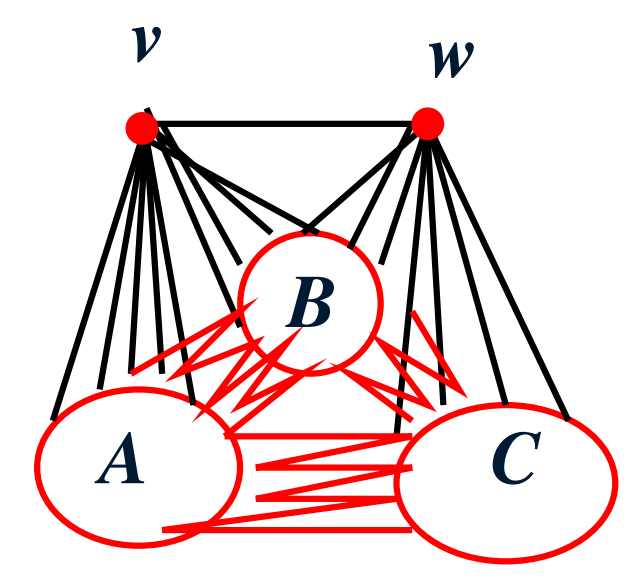

For an edge $u v$ of $G$, the pivoting $u v$ is an operation $G \wedge u v=G * u * v * u$.

O
$H$ is a pivot-minor of $G$ if

$H$ is obtained from $G$ by applying a sequence of pivoting and vertex deletions. 


\section{Tools}

- Isotropic system [Bouchet, 1987] and Scraps

- Extension of Menger's theorem on scraps

- If rank-width of $G$ is $n$, then there is a linked rank-decompositon of width $\boldsymbol{n}$. [Geelen et al., 2002] cf. [Thomas, 1990]

For any $e, f$ in the rank-decomposition $T$, any vertex partition separating $e, f$ has cut-rank $\geq$ min cut-rank of an edge in the path from $e$ to $f$ in $T$.

- Robertson and Seymour's “Lemma on trees" [Robertson and Seymour, 1990] 


\section{Binary matroids and wqo}

Thm (Geelen, Gerards, Whilttle [Geelen et al., 2002]). If $\left\{M_{1}, M_{2}, \ldots\right\}$ is a sequence of binary matroids of branch-width $\leq k$, then there exists $i<j$ such that $M_{i}$ is isomorphic to a minor of $M_{j}$.

Tools

\section{- "Configuration"}

- Extension of Menger's theorem on matroids

- If branch-width of $M$ is $n$, then there is a linked branch-decompositon of width $n$.

For any $e, f$ in the branch-decomposition $T$, any vertex partition separating $e, f$ has connectivity $\geq$ min connectivity of an edge in the path from $e$ to $f$ in $T$.

- Robertson and Seymour's "Lemma on trees"

We generalize this theorem and mimic their proof. 


\section{Our thm implies GGW for binary matroids}

1. For each $M_{i}$, pick a base $B_{i}$ and construct a bipartite graph $G_{i}=$ $\operatorname{Bip}\left(M_{i}, B_{i}\right)$. Branch-width of $M_{i}=$ Rank-width of $G_{i}+1$.

2. Fact: If $H$ is a pivot-minor of $G_{i}$, then there exists a binary matroid $M$ and its base $B$ such that $H=\operatorname{Bip}(M, B)$ and $M$ is a minor of $M_{i}$.

3. [Seymour, 1988] If two binary matroids $M, M^{\prime}$ have the same connectivity function, then $M=M^{\prime}$ or $M=M^{\prime *}$.

If $\operatorname{Bip}\left(M_{i}, B_{i}\right)$ is a vertex-minor of $\operatorname{Bip}\left(M_{j}, B_{j}\right)$ and $M_{i}$ is connected, then $M_{i}$ is a minor of $M_{j}$ or $M_{j}^{*}$.

4. Connected binary matroids of bounded branch-width is wqo. $\exists i<j<k$ such that $\operatorname{Bip}\left(M_{i}, B_{i}\right)$ is isomorphic to a pivot-minor of $\operatorname{Bip}\left(M_{j}, B_{j}\right)$ and $\operatorname{Bip}\left(M_{j}, B_{j}\right)$ is isomorphic to a pivot-minor of $\operatorname{Bip}\left(M_{k}, B_{k}\right)$.

$M_{j}$ is a minor of $M_{k}$ or $M_{i}$ is a minor of $M_{j}$ or $M_{k}$.

5. Apply Higman's lemma to binary matroids. 


\section{Graph and Isotropic system}

We introduce the notion of isotropic systems, defined by [Bouchet, 1987]. The minor of isotropic system is related to the vertex-minor of graphs. The $\alpha \beta$-minor of isotropic system is related to the pivot-minor of graphs. 


\section{Isotropic system}

1. Let $K=\{0, \alpha, \beta, \gamma\}$ be a vector space over $\mathrm{GF}(2)$ with $\alpha+\beta+\gamma=0$.

2. Let $\langle x, y\rangle$ be a bilinear form over $K$. It's uniquely determined; $\langle\boldsymbol{x}, \boldsymbol{y}\rangle=\mathbf{1}$ if $\mathbf{0} \neq \boldsymbol{x} \neq \boldsymbol{y} \neq \mathbf{0},\langle x, y\rangle=0$ otherwise.

3. $K^{V}$ : set of functions from $V$ to $K$. Vector space.

4. For $x, y \in K^{V}$, let $\langle\boldsymbol{x}, \boldsymbol{y}\rangle=\sum_{\boldsymbol{v} \in \boldsymbol{V}}\langle\boldsymbol{x}(\boldsymbol{v}), \boldsymbol{y}(\boldsymbol{v})\rangle \in \mathrm{GF}(2)$. This is a bilinear form.

5. A subspace $L$ is called totally isotropic, if $\langle x, y\rangle=0$ for all $x, y \in L$.

Note: $\operatorname{dim}(L)+\operatorname{dim}\left(L^{\perp}\right)=\operatorname{dim}\left(K^{V}\right)=2|V|$. If $L$ is totally isotropic, $L \subseteq L^{\perp}$.

Def ([Bouchet, 1987]). A pair $S=(V, L)$ is called isotropic system if

- $V$ is a finite set and

- $L$ is a totally isotropic subspace of $K^{V}$ such that $\operatorname{dim}(L)=|V|$. 


\section{Graph $\Rightarrow$ Isotropic system}

For $x \in K^{V}$ and $P \subseteq V, x[P] \in K^{V}$ such that

$$
x[P](v)= \begin{cases}x(v) & \text { if } v \in P \\ 0 & \text { otherwise }\end{cases}
$$

Let $G$ be a graph and $n(v)$ be the set of neighbors of $v$.

Let $a, b \in K^{V}$ such that $a(v), b(v) \neq 0$ for all $v$ and $a(v) \neq b(v)$.

$L$ is a vector space spanned by $\{a[n(v)]+b[\{v\}]: v \in V\}$. Then, $S=(V, L)$ is an isotropic system.

We call $(G, a, b)$ the graphic presentation of $S$. 


\section{Isotropic System $\Rightarrow$ Graph}

$a \in K^{V}$ is called Eulerian vector of $S=(V, L)$, if $a(v) \neq 0$ for all $v \in V$ and $a[P] \notin L$ for all $\emptyset \neq P \subseteq V$.

[Bouchet, 1988] showed

1. There exists an Eulerian vector for any isotropic system.

2. Let $a$ be an Eulerian vector of $S=(V, L)$. For each $v$, there exists a unique vector $b_{v} \in L$ such that $b_{v}(v) \neq 0$ for all $v \in V$ and $b_{v}(w)=0$ or $a(w)$ for all $w \neq v$.

$\left\{b_{v}: v \in V\right\}$ is called the fundamental basis of $S$.

The fundamental graph of $S$ is a graph $(V, E)$ where

$$
v, w \text { are adjacent iff } b_{v}(w) \neq 0 \text {. }
$$

$\operatorname{By}\left\langle b_{v}(w), b_{w}(v)\right\rangle=0, b_{v}(w) \neq 0$ iff $b_{w}(v) \neq 0$. 


\section{Local Complementation and Isotropic system}

Let $G$ be a graph. Let $c_{v}=a\left[n_{G}(v)\right]+b[\{v\}]$.

Consider $G^{\prime}=G * x$. Let $a^{\prime}=a+b[\{x\}]$ and $b^{\prime}=a\left[n_{G}(x)\right]+b$.

$$
c_{v}^{\prime}=a^{\prime}\left[n_{G^{\prime}}(v)\right]+b^{\prime}[\{v\}]= \begin{cases}c_{v}+c_{x} & \text { if } v \sim x \\ c_{v} & \text { otherwise }\end{cases}
$$

Let $L^{\prime}$ be a vector space spanned by $\left\{c_{v}^{\prime}\right\}$. Then, $L^{\prime}=L$.

Local complementation of graphs doesnot change the associated isotropic system. 


\section{Minor}

1. For $X \subseteq V, p_{X}: K^{V} \rightarrow K^{X}$ is a canonical projection such that $\left(p_{X}(x)\right)(v)=x(v)$ for $v \in X$.

2. For a subspace $L$ of $K^{V}$ and $v \in V, a \in K-\{0\}$,

$$
\left.L\right|_{a} ^{v}=\left\{p_{V-\{v\}}(x): x \in L, \mathbf{x}(\mathbf{v})=\mathbf{0} \text { or } \mathbf{a}\right\} \subseteq K^{V-\{v\}} .
$$

For $a \in K-\{0\},\left.S\right|_{a} ^{v}=\left(V-\{v\},\left.L\right|_{a} ^{v}\right)$ is called an elementary minor of $S$.

$S^{\prime}$ is a minor of $S$ if $S^{\prime}=\left.\left.S\right|_{a_{1}} ^{v_{1}}\right|_{a_{2}} ^{v_{2}} \ldots||_{a_{k}}^{v_{k}}$ for some $v_{i}, a_{i}$.

$S^{\prime}$ is an $\boldsymbol{\alpha} \boldsymbol{\beta}$-minor of $S$ if $S^{\prime}=\left.\left.\left.S\right|_{a_{1}} ^{v_{1}}\right|_{a_{2}} ^{v_{2}} \cdots\right|_{a_{k}} ^{v_{k}}$ for some $v_{i}, a_{i} \in\{\alpha, \beta\}$. 


\section{Minor and Vertex-Minor}

Thm ([Bouchet, 1988]). Let $G$ be the fundamental graph of $S$.

Let $H$ be the fundamental graph of $\left.S\right|_{x} ^{v}$.

Then, $H$ is localley equivalent to one of $G \backslash v, G * v \backslash v$, or $G \wedge v w \backslash v$.

Cor. If $S^{\prime}$ is a minor of $S$, then the fundamental graph of $S^{\prime}$ is a vertex-minor of the fundamental graph of $S$. 


\section{$\alpha \beta$-Minor and Pivot-Minor}

Thm. Let $(G, a, b)$ be the graphic presentation of $S$ such that $a(v), b(v) \in$ $\{\alpha, \beta\}$ for all $v \in V(G)$.

Let $\left(H, a^{\prime}, b^{\prime}\right)$ be the graphic presentation of $S^{\prime}$ such that $a^{\prime}(v), b^{\prime}(v) \in$ $\{\alpha, \beta\}$ for all $v \in V(H)$.

If $S^{\prime}$ is an $\boldsymbol{\alpha} \boldsymbol{\beta}$-minor of $S$, then $H$ is a pivot-minor of $G$. 


\section{“Actual” Main Theorem}

We state the theorem written in the language of isotropic system. The proof heavily relies on

- combinatorial lemmas on vector space over $\operatorname{GF}(2)$ with form $\langle$,$\rangle ,$

- isotropic system (or "scraps"), 


\section{Isotropic system and wqo}

- Connectivity $\lambda_{S}(X)=|X|-\operatorname{dim}\left(\left.L\right|_{\subseteq X}\right)=$ CUT-RANK $_{G}(X)$.

- Branch-decomposition and branch-width of isotropic systems.

- $S_{1}=\left(V_{1}, L_{1}\right)$ is simply isomorphic to $S=(V, L)$ if there is a bijectioin $\mu: V_{1} \rightarrow V$ such that for any $x \in K^{V}$,

$$
x \in L \text { if and only if } x \cdot \mu \in L_{1} \text {. }
$$

We prove the following.

Thm. If $\left\{S_{1}, S_{2}, \ldots\right\}$ is an infinite sequence of isotropic systems of bounded branch-width, then there exists $i<j$ such that $S_{i}$ is simply isomorphic to an $\alpha \beta$-minor of $S_{j}$.

This implies our theorem about graphs and pivot-minor. 


\section{Scrap}

$P=(V, L, B)$ is a scrap if $V$ is a finite set and

- $L$ is a totally isotropic subspace of $K^{V}$,

- $B$ is an ordered set (sequence) and a basis of $\boldsymbol{L}^{\perp} / \boldsymbol{L}$.

$|B|=\operatorname{dim}\left(L^{\perp} / L\right)=(2|V|-\operatorname{dim}(L))-\operatorname{dim}(L)=2(|V|-\operatorname{dim}(L))$. If $B=\emptyset$, then $(V, L)$ is an isotropic system.

$P_{1}=\left(X, L^{\prime}, B^{\prime}\right)$ is a minor of $P$ if $X=V \backslash\left\{v_{1}, v_{2}, \ldots, v_{k}\right\}, L^{\prime}=$ $\left.L\right|_{x_{1}} ^{v_{1}}||_{x_{2}}^{v_{2}} \cdots||_{x_{k}}^{v_{k}}$, and $\left|B^{\prime}\right|=|B|$ and $B^{\prime}$ is obtained naturally from $B$ by $\cdots$.

$P_{1}=\left(X, L^{\prime}, B^{\prime}\right)$ is a $\boldsymbol{\alpha} \boldsymbol{\beta}$-minor of $P$ if $X=V \backslash\left\{v_{1}, v_{2}, \ldots, v_{k}\right\}$, $L^{\prime}=\left.L\right|_{x_{1}} ^{v_{1}}||_{x_{2}}^{v_{2}} \cdots \mid{ }_{x_{k}}^{v_{k}}$ with $x_{i} \in\{\alpha, \beta\}$, and $\left|B^{\prime}\right|=|B|$ and $B^{\prime}$ is obtained naturally from $B$ by $\cdots$. 


\section{Very Rough Sketch of Proof}

Suppose $\left\{S_{1}, S_{2}, \ldots\right\}$ is not well-quasi-ordered by $\alpha \beta$-minor relation.

Let $F$ be an infinite forest such that each component is the linked branch-decomposition of $S_{i}$. We attach the root vertex to each component. For an edge $e$, let $l(e), r(e)$ be the left/right child edge incident to $e$. We assign a scrap to each edge of $F$ and define a relation $\leq$ on the set of edges of $F$. We make a scrap of $e$ is a sum of scraps of $l(e)$ and $r(e)$.

By applying lemma on trees, we get a sequence $e_{0}, e_{1}, \ldots$ of edges such that $\left\{e_{0}, e_{1}, \ldots\right\}$ is an antichain and $l\left(e_{0}\right) \leq l\left(e_{1}\right) \leq l\left(e_{2}\right) \leq \cdots$ and $r\left(e_{0}\right) \leq r\left(e_{1}\right) \leq r\left(e_{2}\right) \leq \cdots$.

The number of ways to sum 2 scraps is finite $\Rightarrow \exists i<j, e_{i} \leq e_{j}$. Contradiction. 


\section{Many (strange-looking?) lemmas}

- $\left(\left.L\right|_{x} ^{v}\right)^{\perp}=\left.L^{\perp}\right|_{x} ^{v}$.

- If $X \subseteq V$, then $\left(\left.L\right|_{\subseteq X}\right)^{\perp}=\left.L^{\perp}\right|_{X}$.

- $\operatorname{dim}\left(\left.L\right|_{x} ^{v}\right)= \begin{cases}\operatorname{dim}(L) & \text { if } \delta_{x}^{v} \in L^{\perp} \backslash L \\ \operatorname{dim}(L)-1 & \text { otherwise. }\end{cases}$

- (Extension of Menger's theorem) Let $P=(V, L, B)$ be a scrap and $X \subseteq V$. If $\lambda(P)=\lambda\left(\left.L\right|_{\subseteq X}\right)=\min _{X \subseteq Z \subseteq V} \lambda\left(\left.L\right|_{\subseteq Z}\right)$, then there is an ordered set $B^{\prime}$ such that $Q=\left(X,\left.L\right|_{\subseteq X}, B^{\prime}\right)$ is a scrap and an $\alpha \beta$-minor of $P$. 


\section{Sum and Connection type}

- "sum" of scraps

$P=(V, L, B)$ is a sum of $P_{1}=\left(V_{1}, L_{1}, B_{1}\right)$ and $P_{2}=\left(V_{2}, L_{2}, B_{2}\right)$ if $V_{1} \cap V_{2}=\emptyset$ and $V=V_{1} \cup V_{2}$.

The number of distinct sums of $P_{1}$ and $P_{2}$ are finite up to simple isomorphisms (by "connection type" lemma).

- A connection type $C\left(P, P_{1}, P_{2}\right)$ determines $P$ if $P_{1}$ and $P_{2}$ are given. Roughly speaking, it specifies how $B$ and $L$ are made from $B_{1}$ and $B_{2}$.

- The number of connection type is finite if $\lambda(P)=|V|-\operatorname{dim}(L)$ is bounded.

- If $P_{i}$ is an $\left(\alpha \beta\right.$-)minor of $Q_{i}$ for $i=1,2$ and $P$ is the sum of $P_{1}$ and $P_{2}$ and

$Q$ is the sum of $Q_{1}$ and $Q_{2}$.

If $C\left(P, P_{1}, P_{2}\right)=C\left(Q, Q_{1}, Q_{2}\right)$, then $P$ is an $(\alpha \beta$ - $)$ minor of $Q$. 


\section{Excluded vertex-minors for rank-width $\leq k$}

$G$ is an excluded vertex-minor for a class of graphs of rank-width $\leq k$ if

- Rank-width of $G>k$

- Every proper vertex-minor of $G$ has rank-width $\leq k$.

Cor. For fixed $k$, there are only finitely many excluded vertex-minors for a class of graphs of rank-width $\leq k$.

Proof. An excluded vertex-minor has rank-width $k+1$. Let $E$ be the set of excluded vertex-minors. $E$ is well-quasi-ordered by the vertex-minor relation. But, no excluded vertex-minor contains another. So, $E$ is finite.

Note: The above corollary has an elementary proof.[Oum, 2004]

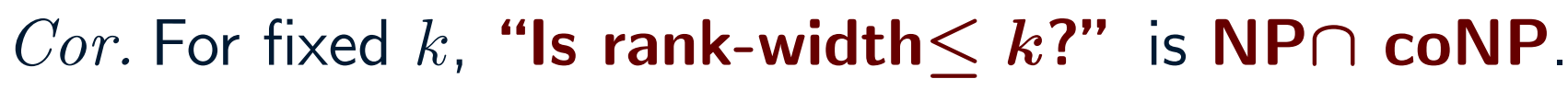

In fact, this is in P. [Courcelle and Oum, 2004] 


\section{References}

[Bouchet, 1987] Bouchet, A. (1987). Isotropic systems. European J. Combin., 8(3):231-244.

[Bouchet, 1988] Bouchet, A. (1988). Graphic presentations of isotropic systems. J. Combin. Theory Ser. B, 45(1):58-76.

[Courcelle and Olariu, 2000] Courcelle, B. and Olariu, S. (2000). Upper bounds to the clique width of graphs. Discrete Appl. Math., 101(1-3):77-114.

[Courcelle and Oum, 2004] Courcelle, B. and Oum, S. (2004). Vertex-minors, monadic second-order logic, and a conjecture by Sesse. submitted.

[Geelen et al., 2002] Geelen, J. F., Gerards, A. M. H., and Whittle, G. (2002). Branch-width and well-quasi-ordering in matroids and graphs. J. Combin. Theory Ser. B, 84(2):270-290.

[Oum, 2004] Oum, S. (2004). Rank-width and vertex-minor. manuscript.

[Oum and Seymour, 2004] Oum, S. and Seymour, P. (2004). Approximating clique-width and branch-width. submitted.

[Robertson and Seymour, 1990] Robertson, N. and Seymour, P. (1990). Graph minors. IV. Tree-width and well-quasi-ordering. J. Combin. Theory Ser. B, 48(2):227-254.

[Seese, 1991] Seese, D. (1991). The structure of the models of decidable monadic theories of graphs. Ann. Pure Appl. Logic, 53(2):169-195.

[Seymour, 1988] Seymour, P. (1988). On the connectivity function of a matroid. J. Combin. Theory Ser. B, 45(1):25-30.

[Thomas, 1990] Thomas, R. (1990). A Menger-like property of tree-width: the finite case. J. Combin. Theory Ser. B, 48(1):67-76. 